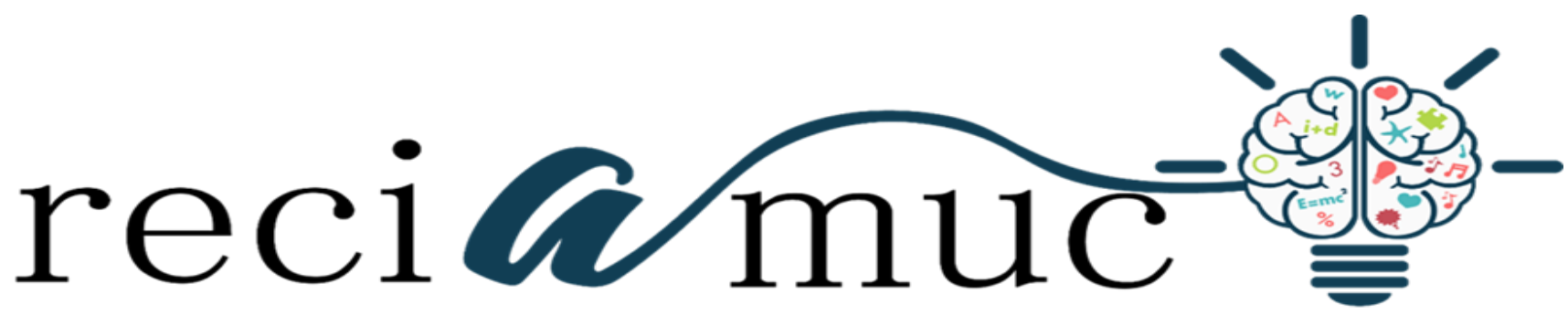

Revista científica de investigación actualización del mundo de las ciencias

Nelson Ricardo Chacha Suscal a; José Luis Campoverde Campoverde b; Mayra Vanessa García Contreras c; Luis Horacio Holguín Carranza d; Paulina Graciela Vallejo Campuzano ${ }^{\mathrm{e}}$; Nelson Oswaldo Zavala Farfán ${ }^{\mathrm{f}}$

Colecistectomía laparoscópica cuidados postoperatorios Laparoscopic cholecystectomy postoperative care

Revista Científica de Investigación actualización del mundo de las Ciencias. Vol. 3 núm., 3, julio. ISSN: 2588-0748, 2018, pp. 1284-1302

DOI: $\underline{\text { 10.26820/reciamuc/3.(3).julio.2019.1284-1302 }}$

URL: http://reciamuc.com/index.php/RECIAMUC/article/view/382 Código UNESCO: 3205 Medicina Interna Tipo de Investigación: Artículo de Investigación (C) RECIAMUC; Editorial Saberes del Conocimiento, 2019

Recibido: $28 / 04 / 2019$ Aceptado: 19/05/2019

Publicado: 01/07/2019

Correspondencia: ricardocsmed93@gmail.com

a. Médico General; Clínica CLIESMETROSA; La Troncal - Ecuador; ricardocsmed93@gmail.com

b. Médico; Clínica CLIESMETROSA; La Troncal - Ecuador; josecampoverde2@ gmail.com

c. Médica General de primer nivel de atención; Administradora técnica del Centro de salud tipo A El Progreso; Nabon-Ecuador; mayagc02@hotmail.com

d. Médico Cirujano; Residente del área de UCI del hospital general IESS; Manta - Ecuador; 1.holguin@ hotmail.com;

e. Médico Cirujano; Investigador Independiente; Manta - Ecuador; paulina_vallejo93@ hotmail.com

f. Médico Cirujano; Médico General en funciones hospitalarias; Hospital General Santo Domingo; Santo Domingo - Ecuador; oswaldo-1992@outlook.es 


\section{Colecistectomía laparoscópica cuidados postoperatorios}

Vol. 3, núm. 3., (2019)

Nelson Ricardo Chacha Suscal; José Luis Campoverde Campoverde; Mayra Vanessa García Contreras; Luis Horacio Holguín Carranza; Paulina Graciela Vallejo Campuzano; Nelson

Oswaldo Zavala Farfán

\section{RESUMEN}

El aparato digestivo se encuentra conformado por órganos huecos como: el tracto gastrointestinal, boca, esófago, estómago, intestino delgado que presenta tres partes, grueso y ano. Mientras los órganos sólidos se encuentran: hígado, páncreas y vesícula biliar, ésta última, cumple la función es la acumulación de bilis, contiene un volumen de alrededor de $50 \mathrm{ml}$ de bilis que libera al duodeno a través de los conductos antes indicados, y entra en él a través de la papila y ampolla de Vater que está adherida a la superficie visceral del hígado. Sin embargo, este órgano es más conocida por los potenciales problemas que puede ocasionar, debido a que sirve de asiento de los cálculos de sales biliares (piedras pequeñas) que obstruyen la vía biliar y generan patologías inflamatorias que en algunos casos, pueden eliminarse con medicamentos, en otros deben tratarse quirúrgicamente, su inflamación es conocida como colecistitis; además, puede presentar tumores. Por ello, una vez diagnosticada la inflamación, el médico tratante recomienda la intervención quirúrgica para remover la vesícula biliar usando un dispositivo médico llamado laparoscopia, durante dicho proceso se realizan entre uno o cuatro pequeñas incisiones en el abdomen procedimiento llamado colecistectomía. En consecuencia, una vez que el paciente es dado de alta, debe cumplir una serie de recomendaciones postoperatorias entre las cuales se encuentran: comer bajo en grasa, ingerir analgésicos o antiinflamatorios para reducir los efectos del dolor, evitar la realización de movimientos para ayudar a la herida a una cicatrización entre otros. Lo expuesto, sirve de aporte para el desarrollo del presente artículo cuyo objetivo es analizar la colecistectomía laparoscópica cuidados postoperatorios, apoyada en una investigación documental para luego construir las conclusiones del mismo.

Palabras Claves: Colecistectomía Laparoscópica; Cuidados Postoperatorios. 


\section{Colecistectomía laparoscópica cuidados postoperatorios}

Vol. 3, núm. 3., (2019)

Nelson Ricardo Chacha Suscal; José Luis Campoverde Campoverde; Mayra Vanessa García

Contreras; Luis Horacio Holguín Carranza; Paulina Graciela Vallejo Campuzano; Nelson

Oswaldo Zavala Farfán

\section{ABSTRACT}

The digestive tract is composed of hollow organs such as: gastrointestinal tract, mouth, esophagus, stomach, small intestine presenting three parts, thickness and year. While the solid organs are: liver, pancreas and gallbladder, the latter, the role is the accumulation of bile, it contains a volume of about $50 \mathrm{ml}$ of bile that released into the duodenum through the above lines, and enters it through the papilla and ampulla of Vater which is attached to the visceral surface of the liver. However, this body is more known for the potential problems that can cause, since it serves as seat of the calculations of bile salts (small stones) that obstruct the bile and generate inflammatory diseases that in some cases, can eliminated with medication, in others they should be treated surgically, its inflammation is known as cholecystitis; You can also submit tumors. Therefore, once diagnosed inflammation, the doctor recommended surgery to remove the gallbladder using a medical device called a laparoscopy, during this process are made between one or four small incisions in the abdomen procedure called cholecystectomy. As a result, a time that the patient is discharged, must meet a number of postoperative recommendations which include: eating low fat, ingested analgesics or anti-inflammatory drugs to reduce the effects of pain, prevent the realization of movements to help the wound to one healing among others. Serves as a contribution for the development of this article whose aim is analyzing the laparoscopic cholecystectomy postoperative care, supported by a documentary research to then build the conclusions thereof.

Key Words: Laparoscopic cholecystectomy; Postoperative care. 


\section{Colecistectomía laparoscópica cuidados postoperatorios}

Vol. 3, núm. 3., (2019)

Nelson Ricardo Chacha Suscal; José Luis Campoverde Campoverde; Mayra Vanessa García Contreras; Luis Horacio Holguín Carranza; Paulina Graciela Vallejo Campuzano; Nelson

Oswaldo Zavala Farfán

\section{Introducción.}

La extracción mínimamente invasiva de la vesícula biliar (colecistectomía laparoscópica) es el tratamiento de elección actualmente para los pacientes con síntomas relacionados con cálculos en la vesícula biliar. Este procedimiento se realiza mediante la distensión del abdomen con dióxido de carbono (neumoperitoneo) para que haya un espacio adecuado para los instrumentos y visualizar las estructuras dentro del abdomen. Lo anterior permite a los cirujanos identificar y dividir las estructuras apropiadas.

Sin embargo, la distensión de la pared abdominal puede dar lugar a diversos cambios fisiológicos que afectan el funcionamiento del corazón o pulmones. Estos cambios son más pronunciados al utilizar presiones mayores de gas para distender el abdomen. Por lo general, son bien tolerados en pacientes con un bajo riesgo de problemas anestésicos. Mientras que en aquellos pacientes con enfermedades preexistentes pueden no tolerar bien esta distensión del abdomen. Por lo tanto, se recomienda un método alternativo que permitir a los cirujanos visualizar las estructuras en el abdomen y utilizar los instrumentos mediante el levantamiento de la pared abdominal con dispositivos especiales (elevación de la pared abdominal) en pacientes sometidos a la colecistectomía laparoscópica.

En relación a este último planteamiento, Matos (2016) precisa que mediante la colecistectomía "se extirpa la vesícula biliar y los cálculos biliares a través de varios cortes (incisiones) en el abdomen. El cirujano le inflará el abdomen con aire o dióxido de carbono para ver con claridad.” (p.25). Es decir, la función que caracteriza a esta intervención quirúrgica es lograr la respectiva extirpación de la vesícula biliar, con ello, se logra eliminar también los cálculos biliares presente y mejorar la calidad de vida del paciente.

Cabe agregar, que, una vez realizada la extirpación de la vesícula biliar, la bilis va a fluir desde el hígado (donde se produce) a través del conducto colédoco y hasta el intestino delgado. Debido a que se ha extirpado la vesícula biliar, pues, el cuerpo no puede almacenar bilis entre comidas. A la mayoría de las personas esto les causa pocos o ningún efecto digestivo. De igual manera, Matos (ob.cit) destaca que "el 98,35\% de la colecistectomía laparoscópica fue programada, solo 1,64\% 


\section{Colecistectomía laparoscópica cuidados postoperatorios}

Vol. 3, núm. 3., (2019)

Nelson Ricardo Chacha Suscal; José Luis Campoverde Campoverde; Mayra Vanessa García Contreras; Luis Horacio Holguín Carranza; Paulina Graciela Vallejo Campuzano; Nelson Oswaldo Zavala Farfán

urgente, por vía convencional de forma urgente $(79,13 \%)$, lo cual se debió a que en estos pacientes tenían alguna causa que contraindicaba la vía laparoscópica”. (p.27).

Según la apreciación citada, se puede entender que, mediante la realización de dicho proceso quirúrgico, el paciente tiene oportunidades más amplias de una recuperación rápida y eficaz, además, la misma mantiene una tendencia significativa en cuanto a su realización, pues, sus riesgos son mínimos en comparación con la vía convencional; no obstante, en casos adversos no se programa para ser efectuada. Tal como lo indica, Matos (ob.cit), "la mortalidad global de la colecistectomía laparotómica fue de 0,39\% y de la laparoscópica de 0,07\%. (p.27). Resultados que llevan a reflejar un nivel bajo de mortalidad, para deducir que mediante esta intervención los pacientes lograr tener menores riesgos.

En consecuencia, una vez llevado a cabo la intervención quirúrgica, el paciente debe cumplir con un reposo postoperatorio, mediante el cual lograr su recuperación total, pero durante el mismo, es importante mantener y cumplir las respectivas recomendaciones dadas por el especialista tratante. Al respecto, Rangel (2018), destaca que después de una colecistectomía laparoscópica “es importante reducir las grasas, aumentar la fibra, comer porciones pequeñas con frecuencia, reducir los lácteos entre otros". (p.8). De allí, que para lograr un postoperatorio favorable para el paciente, es importante considerar, los alimentos a consumir y el nivel de grasa, pues, al carecer de la vesícula biliar, les corresponde a los intestinos, esto puede generar un efecto laxante, aunado a gases e hinchazón al permanecer mayor tiempo para digerir la cantidad de grasa ingerida.

Cada una de las consideraciones descritas en párrafos anteriores, son los encargados de estimar el desarrollo de este artículo, mediante el cual, se busca llegar a un análisis, preciso, coherente y exhaustivo en cuanto al tema a tratar, para luego precisar de forma amplia las conclusiones generales que servirán para tener una visión global de los aspectos relevantes insertados en su contenido. 


\section{Colecistectomía laparoscópica cuidados postoperatorios}

Vol. 3, núm. 3., (2019)

Nelson Ricardo Chacha Suscal; José Luis Campoverde Campoverde; Mayra Vanessa García Contreras; Luis Horacio Holguín Carranza; Paulina Graciela Vallejo Campuzano; Nelson

Oswaldo Zavala Farfán

\section{Método.}

Para mantener la operatividad metodológica del presente artículo, se determina la selección del método como herramienta esencial encargada de proporcionar visiones generales en relación al contenido tratado, para lo cual, es importante considerar la definición dada por, Ventura (2018) los métodos de investigación "son aquellos que le aseguran al investigador la obtención del conocimiento desde una visión global de los hechos". (p.69). Por lo tanto, la escogencia del camino para reflexionar en cuanto a los diferentes tópicos esenciales que dan cabida a la construcción de un cuerpo interpretativo y de apoyo documental para este proceso investigativo, se estima la selección del inductivo.

Al establecer como método el tipo inductivo, significa que las indagaciones de los distintos contenidos fueron valoradas desde una visión individual, para luego conjugarlos y trasladar las interpretaciones a contexto global, de esta forma se diseñan las apreciaciones enmarcadas en aquellas situaciones que forman parte del fenómeno en estudio. En tal sentido, Ventura (ob.cit) destaca que el método inductivo "lleva a leer, interpretar, separar y complementar con las experiencias del investigador para generar nuevas visiones vinculadas con un tema previamente seleccionado". (p.71).

\section{Tipo de Investigación.}

La continuidad del proceso investigativo, permite tener el respectivo acercamiento a la identificación de su tipo en correspondencia con un nivel que según Arias (2012) "se refiere al grado de profundidad con que se aborda un objeto o fenómeno" (p.45). Por lo tanto, el desarrollo de esta actividad, lleva a resaltar que, debido a estar en correspondencia con el tipo documental, su contenido estará estimado por un nivel donde se logra no sólo interpretar los argumentos básicos del fenómeno, sino que se amplían sus valoraciones para así generar nuevas valoraciones significativas e importantes para otra actividad investigativa

En función a lo anterior, se puede indicar que toda investigación documental de acuerdo con Sandoval (2015) "representa el estudio de un problema con el propósito de ampliar y profundizar 


\section{Colecistectomía laparoscópica cuidados postoperatorios}

Vol. 3, núm. 3., (2019)

Nelson Ricardo Chacha Suscal; José Luis Campoverde Campoverde; Mayra Vanessa García Contreras; Luis Horacio Holguín Carranza; Paulina Graciela Vallejo Campuzano; Nelson Oswaldo Zavala Farfán

el conocimiento de su naturaleza con apoyo en trabajos previos divulgados por medios impresos o electrónicos" (p.60). De allí, que se estimaron una serie de actividades centradas en lograr recabar las informaciones previas que dieron como resultado, la respectiva elaboración del análisis relacionado con el fenómeno en estudio.

\section{Fuentes Documentales.}

Cada una de las consideraciones expuestas en párrafos anteriores, permiten comprender que la conducción efectiva del proceso investigativo, necesita la presencia de herramientas básicas que ayuden a la selección adecuada de los aspectos relativos al fenómeno en estudio, para ello, se incorporaron aquellos medios esenciales que dan cabida a la organización del texto. En esta dirección Sandoval (ob.cit) las define "como la suma de medios especiales que están directamente vinculados con el tipo de investigación y se incorporar para recopilar la información previa” (p.65). En consecuencia, para el desarrollo del artículo, se tuvo que adecuar las herramientas de lecturas rápida, reflexiva, el fichaje, organización de textos entre otras fuentes documentales.

\section{Técnicas para la Recolección de la Información.}

El desarrollo del contenido que caracteriza a este artículo, se encuentra determinado por la necesidad de incluir en su realización diferentes técnicas viables que dieron como resultado una recopilación importante y de interés para dar continuidad al fenómeno a indagar, tal como lo expresa Sandoval (ob.cit) las técnicas de recolección de información "sirven de apoyo para la verificación de un contenido en particular y hace posible reconocer los diferentes elementos que lo representan en un momento determinado".(p.66). Por ello, se incorporó la lectura rápida mediante la cual, se pudo hacer un arqueo de las referencias más importantes para ampliar los contenidos seleccionados, asimismo, se llevó a cabo el fichaje mediante el cual, se pudo estimar la realidad de las apreciaciones dadas por los teóricos desde una perspectiva global y construir definitivamente los argumentos básicos que dieron cabida a la ampliación del contenido propuesto. 


\section{Colecistectomía laparoscópica cuidados postoperatorios}

Vol. 3, núm. 3., (2019)

Nelson Ricardo Chacha Suscal; José Luis Campoverde Campoverde; Mayra Vanessa García Contreras; Luis Horacio Holguín Carranza; Paulina Graciela Vallejo Campuzano; Nelson

Oswaldo Zavala Farfán

\section{Resultados.}

La visión científica que caracteriza a todo proceso investigativo, debe estar encaminado hacia la búsqueda de información pertinente, en este particular, se consideraron los eventos que forman parte del artículo, es decir, se fijaron los aspectos en función a sus propias características que determinan el fenómeno en estudio.

\section{Anatomía y Fisiología de la Vesícula Biliar.}

La vesicular biliar funciona como el reservorio de bilis localizada en la cara inferior del hígado en la confluencia de los dos lóbulos hepáticos o línea de Cantlie entre los segmentos IVb y V. En $50 \%$ de los casos se encuentra a nivel del noveno cartílago costal. Es un saco en forma de pera, y en un adulto mide cerca de $10 \mathrm{~cm}$ de longitud, 3 a $4 \mathrm{~cm}$ de ancho con una pared de 1 a $2 \mathrm{~mm}$. Su capacidad es de 40 a $70 \mathrm{ml}$, pero puede llegar a ser de $100 \mathrm{ml}$. Se separa del parénquima hepático por una capa fina de tejido conjuntivo derivado de la cápsula de Glisson o puede estar adherida al parénquima. Por lo general, se encuentra en la fosa vesicular; sin embargo, es factible que su ubicación sea intrahepática, extraperitoneal, en el omento menor, en el ligamento falciforme, inferior al diafragma derecho y en contadas ocasiones en el hiato de Winslow.

En consecuencia, la vesícula biliar se encuentra rodeada de peritoneo visceral y está en contacto con el píloro, duodeno, cabeza de páncreas y flexura hepática del colon; varía en tamaño, se divide en fondo, cuerpo, infundíbulo y cuello con o sin bolsa de Hartmann; se cree que es consecuencia de una inflamación crónica más que a una estructura anatómica en 2 a $6 \%$ de los casos. El fondo llega al borde libre del hígado o lo puede rebasar, el cuerpo es la parte más grande, y el infundíbulo es la conexión entre el cuerpo y cuello. Cabe agregar que, se une a la vía biliar a través del conducto cístico, continuando junto con el conducto hepático en el colédoco y desembocando en el duodeno a través de la ampolla de Vater, que funciona a modo de esfínter. La arteria cística, rama de la arteria hepática propia, irriga la vesícula biliar y el conducto cístico. Acompaña al conducto cístico en su entrada a la vesícula. 


\section{Colecistectomía laparoscópica cuidados postoperatorios}

Vol. 3, núm. 3., (2019)

Nelson Ricardo Chacha Suscal; José Luis Campoverde Campoverde; Mayra Vanessa García Contreras; Luis Horacio Holguín Carranza; Paulina Graciela Vallejo Campuzano; Nelson Oswaldo Zavala Farfán

En cuanto a su función es acumular la bilis producida por el hígado hasta el momento de la digestión, donde se verterá el contenido de la vesícula en el tubo digestivo. La bilis es un líquido de color verde oscuro que tiene la función de emulsionar las grasas, facilitando así su digestión y absorción, y de favorecer los movimientos intestinales. Además, a través de la bilis se excretan el exceso de colesterol y productos de desecho del metabolismo de la hemoglobina, como labilirrubina, además de algunos medicamentos. La bilis secretada por el hígado llega a la vesícula a través del conducto hepático y cístico, hasta que el estímulo de la digestión hace que se contraiga la pared muscular de la vesícula y expulse la bilis. La secreción de la bilis está estimulada por la ingesta de alimentos, sobre todo carne o grasas. La vesícula puede almacenar hasta $50 \mathrm{ml}$ de bilis que libera al duodeno en el momento de la digestión.

Figura $\mathbf{N}^{\circ} 1$ Anatomía de la Vesícula Biliar

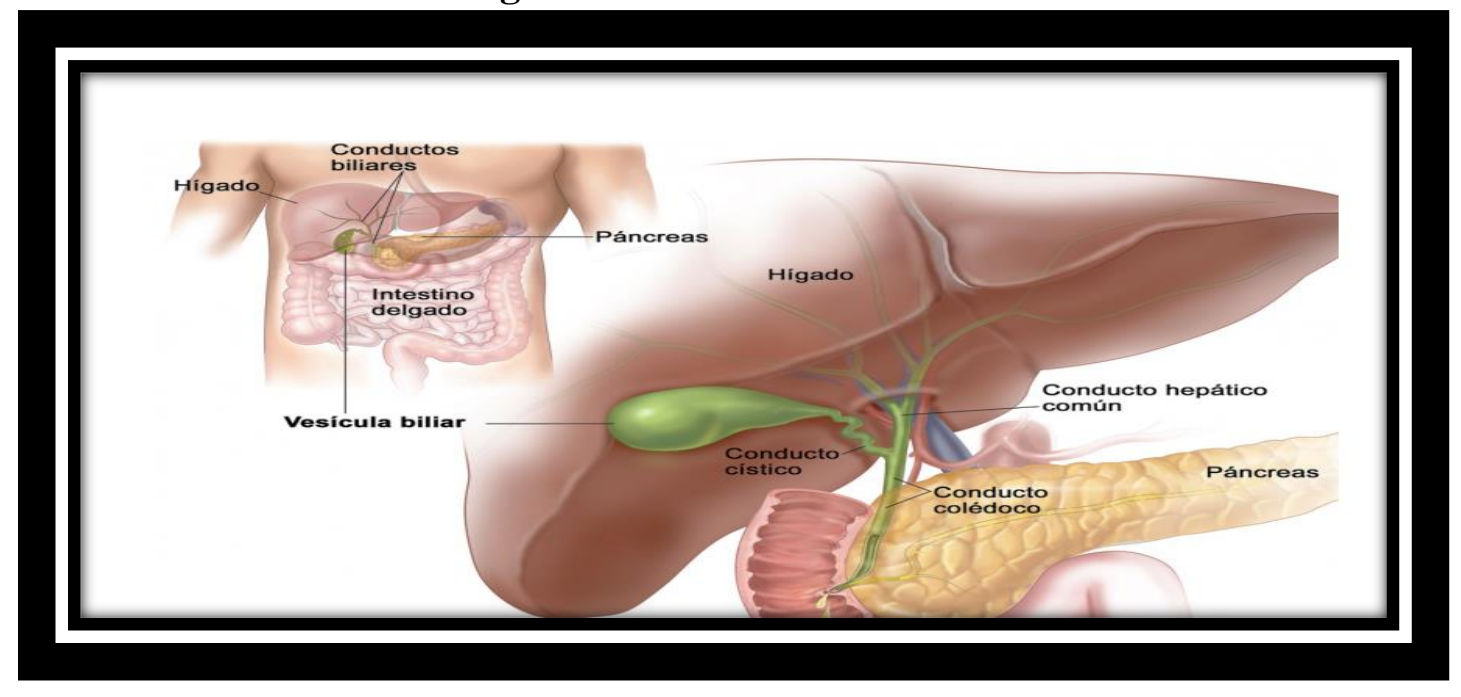

Fuente: Matos (ob.cit)

La vesícula biliar es una bolsa de 7 a 10cm de longitud y de 3 a $5 \mathrm{~cm}$ de ancho, la cual mantiene en promedio un volumen de 30-50ml; tiene forma piriforme, se divide para su estudio en tres secciones: fondo, cuerpo y cuello; en la región del cuello puede haber un infundíbulo, que se denomina bolsa de Hartmann. La misma, se encuentra dispuesta en el borde inferior del hígado, entre el lóbulo derecho e izquierdo, la vena hepática media se sitúa en el mismo plano anatómico y puede utilizarse para ayudar a encontrar la fosa vesicular. 


\section{Colecistectomía laparoscópica cuidados postoperatorios}

Vol. 3, núm. 3., (2019)

Nelson Ricardo Chacha Suscal; José Luis Campoverde Campoverde; Mayra Vanessa García Contreras; Luis Horacio Holguín Carranza; Paulina Graciela Vallejo Campuzano; Nelson

Oswaldo Zavala Farfán

Asimismo, las vías biliares se dividen en dos tipos, las vías intrahepaticas y extrahepaticas; las primeras tienen su origen en los canalículos biliares y vierten en los conductillos interlobulillares, la segunda está compuesta por los conductos biliares principales los cuales se dividen en conducto hepático derecho e izquierdo los cuales emergen del hilio hepático, en la unión de los conductos hepáticos derecho e izquierdo se continua uno solo, denominado conducto hepático común. Del conducto hepático común se deriva otro que se dirige hacia la vesícula biliar, denominado conducto cístico, a partir de ese punto, el conducto hepático común cambia de nombre a conducto colédoco, el cual se unirá en la región duodenal con el conducto de Wirsung o también conocido como conducto pancreático.

Figura $\mathbf{N}^{\circ} 2$ Vesícula Biliar

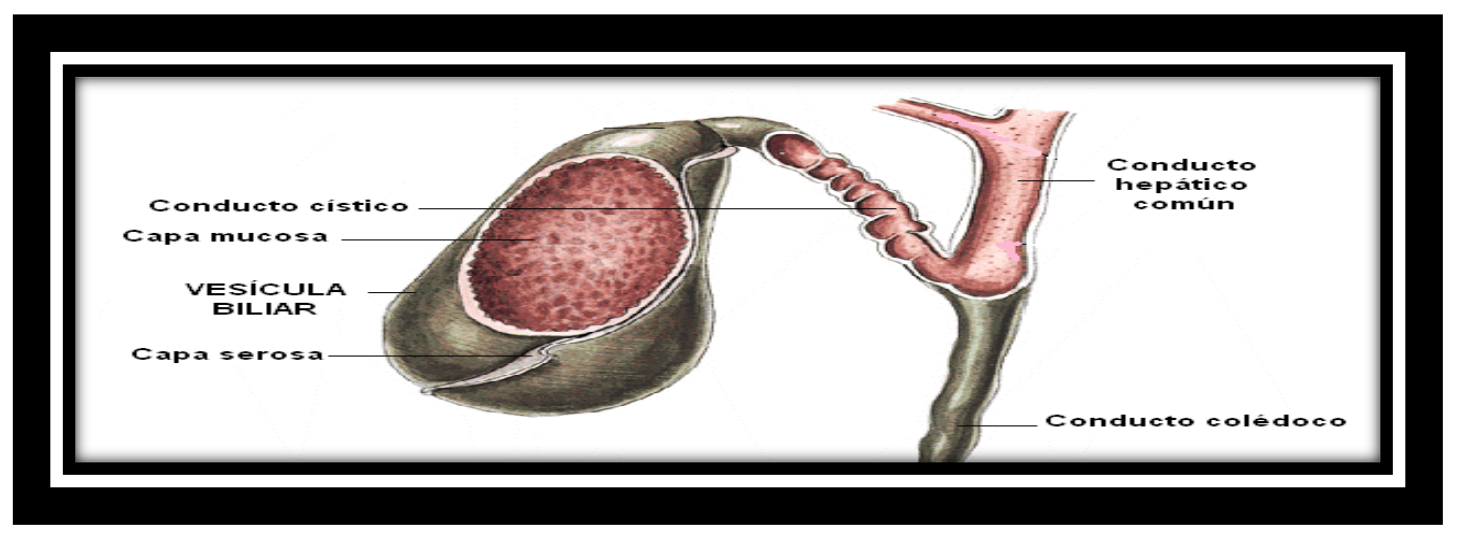

Fuente: Matos (ob.cit)

Es importante acotar que, la vesícula biliar puede provocar enfermedades, normalmente en las situaciones en las que se retrasa o se obstruye el flujo de la bilis. La patología biliar más común en la colelitiasis, que consiste en la producción de cálculos de sales biliares que pueden obstruir la salida de bilis a la vía biliar. El grado de obstrucción condicionará el tipo de patología. Una obstrucción intermitente puede derivar en cólicos hepáticos, con dolores intermitentes después de las comidas sin asociar otros síntomas. Cuando la obstrucción además suponga una inflamación de las paredes de la vesícula, estaremos ante una colecistitis, que supone generalmente un dolor continúo asociado a náuseas, vómitos y fiebre. En otros casos, las vías biliares además de 


\section{Colecistectomía laparoscópica cuidados postoperatorios}

Vol. 3, núm. 3., (2019)

Nelson Ricardo Chacha Suscal; José Luis Campoverde Campoverde; Mayra Vanessa García Contreras; Luis Horacio Holguín Carranza; Paulina Graciela Vallejo Campuzano; Nelson Oswaldo Zavala Farfán

obstruirse, pueden ser asiento de tumores. En la mayor parte de los casos, la patología biliar ha de ser tratada quirúrgicamente mediante la extirpación de la vesícula biliar.

\section{Colecistectomía de la Vesícula Biliar.}

La colecistectomía es un procedimiento quirúrgico para extirpar la vesícula biliar, un órgano con forma de pera que se encuentra justo debajo del hígado, en la parte superior derecha del abdomen. La vesícula biliar recoge y almacena la bilis, un jugo digestivo producido en el hígado. Puede ser necesaria si presentas dolor a causa de cálculos biliares que bloquean el paso de la bilis. La colecistectomía es una cirugía frecuente que conlleva un riesgo muy bajo de tener complicaciones. En la mayoría de los casos, podrás volver a tu casa el mismo día de la colecistectomía.

En la mayoría de los casos, se realiza mediante la introducción de una cámara de vídeo diminuta y herramientas quirúrgicas especiales a través de cuatro incisiones pequeñas para poder ver el interior del abdomen y extirpar la vesícula biliar. Los médicos llaman a este procedimiento «colecistectomía laparoscópica. En algunos casos, se realizará una gran incisión para extirpar la vesícula biliar. Este procedimiento se llama colecistectomía abierta. Por lo general, las colecistectomías se realizan para tratar los cálculos biliares y las complicaciones que causan. El médico puede recomendar una colecistectomía si padece de: cálculos biliares en la vesícula (colelitiasis), cálculos biliares en las vías biliares (coledocolitiasis), inflamación de la vesícula (colecistitis) e inflamación del páncreas (pancreatitis) debido a los cálculos biliares.

Por ello, los pacientes que son sometidos a una colecistectomía laparoscópica se va a la casa el día de la cirugía o al otro día y reanuda su dieta y sus actividades normales de inmediato. En cambio, cuando se recurre a la colecistectomía abierta requiere de cinco a siete días de hospitalización y reanuda su dieta normal luego de una semana. Las actividades normales se reanudan entre cuatro y seis semanas después de la cirugía. A la hora de la disección de la vesícula, es fundamental definir el triángulo de Calot para realizar una disección segura y evitar una lesión de la vía biliar. El límite superior del triángulo es la arteria cística, el límite inferior es el conducto cístico y la cara medial está formada por el conducto hepático. 


\section{Colecistectomía laparoscópica cuidados postoperatorios}

Vol. 3, núm. 3., (2019)

Nelson Ricardo Chacha Suscal; José Luis Campoverde Campoverde; Mayra Vanessa García Contreras; Luis Horacio Holguín Carranza; Paulina Graciela Vallejo Campuzano; Nelson Oswaldo Zavala Farfán

Es importante acotar, que la decisión de realizar este tipo de intervención quirúrgica, se encuentra determinado cuando surgen problemas de la vesícula biliar, que son usualmente causados por la presencia de cálculos, es decir, son pequeñas piedras compuestos principalmente de colesterol y sales biliares, formados en la vesícula o en la vía biliar. Hasta hoy, no se sabe porque algunas personas forman cálculos, tampoco se conocen alguna medida para prevenir los cálculos, su presencia puede obstruir la salida de la bilis de la vesícula, causando su inflamación, dolor abdominal agudo, vómito, indigestión, y ocasionalmente, fiebre. Si el cálculo obstruye el colédoco, se puede producir ictericia (color amarillo en la piel).

Por lo tanto, la extracción laparoscópica de la vesícula biliar, se hace bajo anestesia general, el paciente va estar dormido durante la cirugía. Usando una cánula (tubo delgado) el cirujano entra al abdomen en la región del ombligo. Una laparoscopia (un fino telescopio) conectado a una cámara especial es introducido a través de la cánula, dando al cirujano una imagen magnificada de los órganos internos del paciente sobre una pantalla de televisión. Otras cánulas son insertadas para permitirle a su cirujano separar delicadamente la vesícula de sus adherencias y extraerla a través de una de las aperturas. Muchos cirujanos realizan una radiografía, llamada colangiografía, para identificar cálculos los cuales pueden estar localizados en la vía biliar o asegurar que las estructuras anatómicas han sido identificadas. Si el cirujano encuentra uno o más cálculos en el colédoco, puede extraerlos usando un endoscopio especial, puede decidir extraerlos después usando otro procedimiento mínimamente invasivo o puede convertirla a una cirugía abierta con el objeto de extraer todos los cálculos en esta cirugía. Después de que el cirujano ha extraído la vesícula, las pequeñas incisiones son cerradas con un punto o dos, o con una cinta quirúrgica.

En este orden de ideas, Dávila (2017), resalta una serie de consideraciones significativas en cuanto a resultados vinculados con la colecistectomía laparoscópica de la vesícula biliar entre ellos:

En la colecistectomía, un $80 \%$ fueron mujeres $(80 \%)$ y (20\%) hombres. En edades entre los 18 y 76 años, con una media de 47 años. Durante el período preoperatorio un (42,5\%) de pacientes estaban asintomáticos y (57,5\%) sintomáticos. Entre los pacientes sintomáticos, los síntomas preoperatorios más prevalentes fueron 


\section{Colecistectomía laparoscópica cuidados postoperatorios}

Vol. 3, núm. 3., (2019)

Nelson Ricardo Chacha Suscal; José Luis Campoverde Campoverde; Mayra Vanessa García Contreras; Luis Horacio Holguín Carranza; Paulina Graciela Vallejo Campuzano; Nelson Oswaldo Zavala Farfán

estreñimiento y flatulencia. Estos aparecieron solos, juntos o con otros síntomas. Considerando a los pacientes asintomáticos antes de la operación los que tenían una dieta baja en grasa, el $(63,6 \%)$ no presentaron síntomas digestivos después de la operación, y solo $(36,4 \%)$ lo manifestaron. En cambios el $(66,7 \%)$ que tenía dieta normal continuaron sin presentar síntomas digestivos después de la operación y $(33,3 \%)$ aparecieron síntomas de inicio. (p.36)

Según los hallazgos presentados por Dávila, permite deducir, que los pacientes intervenidos por esta técnica innovadora, dejaron de sentir los síntomas previos al proceso quirúrgico, asimismo, se evidencia que aquellos sometidos a una dieta normal continuaron sin registrar síntomas digestivos, aspecto de significación para los pacientes, pues, su recuperación, se hace con mayor rapidez y puede incorporarse de manera más eficiente a la vida normal. Estos resultados muestran la ausencia de un efecto significativo de la dieta baja en grasas en la prevención o reducción de los síntomas dispépticos en el período postoperatorio inmediato de la colecistectomía laparoscópica; por lo tanto, la prescripción de una dieta baja en grasa dependerá de la línea que el cirujano prefiera seguir y perfil del paciente, sus condiciones clínicas asociadas, dado que, algunos prefieren mantener la dieta preoperatoria baja en grasas después de la colecistectomía debido a factores psicológicos.

Dentro de la misma línea de ideas, la colecistitis ocurre cuando la vesícula se inflama, según Rojas (2017), entre sus causas pueden ser:

Cálculos biliares: Con mayor frecuencia, la colecistitis se produce a causa de partículas duras que se forman en la vesícula (cálculos biliares). Los cálculos biliares pueden obstruir el tubo (conducto cístico) por el que fluye la bilis cuando sale de la vesícula. La bilis se acumula y causa la inflamación.

Tumor: Un tumor puede evitar que la bilis drene de la vesícula de forma adecuada, lo que causa la acumulación de bilis que puede provocar colecistitis.

Obstrucción de las vías biliares: La torsión o la formación de cicatrices de las vías biliares pueden causar obstrucciones que provoquen colecistitis. 


\section{Colecistectomía laparoscópica cuidados postoperatorios}

Vol. 3, núm. 3., (2019)

Nelson Ricardo Chacha Suscal; José Luis Campoverde Campoverde; Mayra Vanessa García Contreras; Luis Horacio Holguín Carranza; Paulina Graciela Vallejo Campuzano; Nelson Oswaldo Zavala Farfán

Infecciones: El sida y ciertas infecciones virales pueden ocasionar la inflamación de la vesícula.

Problemas en los vasos sanguíneos: Una enfermedad muy grave puede dañar los vasos sanguíneos y disminuir el flujo de sangre hacia la vesícula, lo que da lugar a la colecistitis.

El autor, destaca que la colecistitis puede ocasionar una serie de complicaciones graves, entre ellas:

Infección en la vesícula: $\mathrm{Si}$ se acumula bilis en la vesícula, lo que provoca colecistitis, esta se puede infectar.

Muerte del tejido de la vesícula: Si la colecistitis no se trata, puede provocar la muerte del tejido de la vesícula (gangrena). Es la complicación más frecuente, especialmente, en las personas mayores, las que tienen diabetes y quienes esperan para recibir tratamiento. Esto puede provocar un desgarro o una rotura en la vesícula.

Desgarro de la vesícula: Un desgarro (una perforación) en la vesícula se puede producir a partir de una inflamación, una infección o la muerte del tejido de la vesícula.

Figura $\mathbf{N}^{\circ} 3$ Colecistectomía Laparoscópica

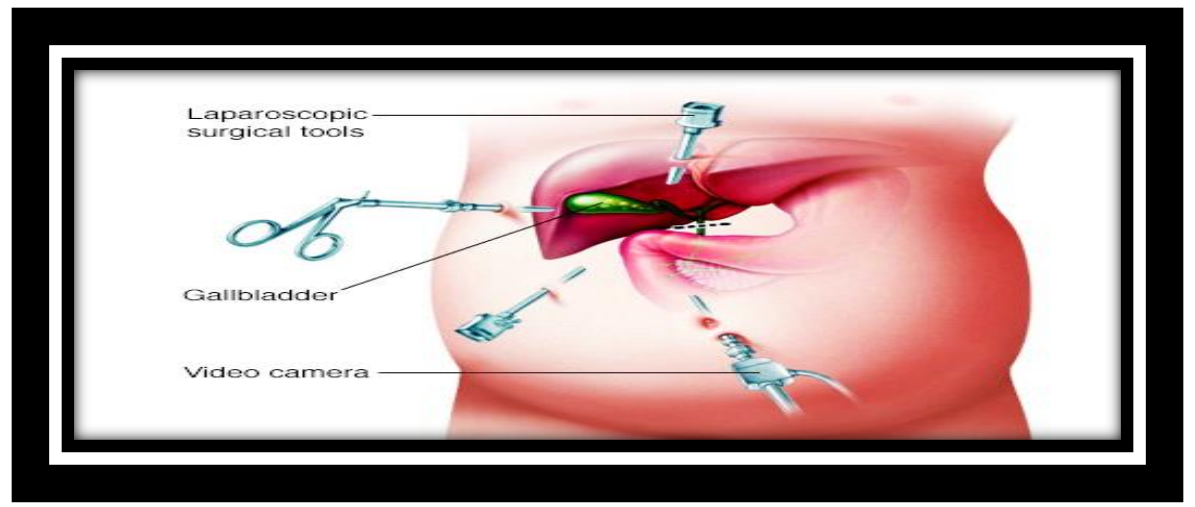

Fuente: Dávila (ob.cit)

Del análisis precedente, se puede indicar que para lograr una recuperación efectiva del paciente, hay que tener un cuidado postoperatorio funcional, que ayude a regular las funciones biliares, pues, una vez extirpada la vesícula, el proceso biliar se hace a través de los intestinos, situación que trae 


\section{Colecistectomía laparoscópica cuidados postoperatorios}

Vol. 3, núm. 3., (2019)

Nelson Ricardo Chacha Suscal; José Luis Campoverde Campoverde; Mayra Vanessa García Contreras; Luis Horacio Holguín Carranza; Paulina Graciela Vallejo Campuzano; Nelson Oswaldo Zavala Farfán

consecuencias, como diarreas hinchazón del abdomen, dolor agudo, todo ello, debido a la presencia de los cálculos biliares, para así producirse la colecistitis que no es más que una infamación de la vesícula, que contiene líquido digestivo (bilis) y libera al intestino delgado. En su mayoría la colecistitis se produce por cálculos biliares que obstruyen el tubo que sale de la vesícula, para así generar una acumulación de bilis que puede causar inflamación, también puede deberse a problemas con el conducto biliar, tumores, enfermedades graves y ciertas infecciones, que al no ser tratadas producen complicaciones graves que, pueden poner en peligro la vida, como la rotura de la vesícula, el tratamiento de la colecistitis suele consistir en la extirpación de la vesícula.

\section{Cuidados Postoperatorios de la Colecistectomía Laparoscópica de Vesícula Biliar.}

La cirugía para extirpar la vesícula biliar se llama colecistectomía. Durante la cirugía tradicional, se extrae la vesícula biliar a través de una incisión (corte) de 5 a 8 pulgadas de largo en el abdomen, llamada colecistectomía abierta. Sin embargo, la forma más común de extirpar la vesícula biliar es mediante la colecistectomía laparoscópica. Durante esta cirugía, se realizan de 3 a 4 pequeñas incisiones (cortes) en el vientre. Luego, se insertan instrumentos por las incisiones. El médico cirujano utiliza los instrumentos para mirar la vesícula biliar y extraerla. Después de extirpada la vesícula biliar, se procede a bloquear todos los conductos biliares y cerrará las incisiones con puntadas, grapas o pegamento. Este procedimiento tarda de 1 a 2 horas. La mayoría de las personas se van a casa el mismo día o el día después de la cirugía.

En este orden de ideas, se puede decir que, mediante la realización de este proceso quirúrgico, se puede realizar sin cortar los músculos del abdomen, lo que permite tener diferentes ventajas entre ellas: Tener menos dolor después de la cirugía. Estar internado en el hospital menos tiempo. Tener un tiempo de recuperación más corto. Regresar al trabajo más rápido. Tienen cicatrices mucho menos notables que con una colecistectomía tradicional. Es decir, está técnica quirúrgica es más cómoda para llevar a cabo la operación de extracción de vesícula, puesto que reduce la estancia hospitalaria, tiempo de recuperación y tamaño de las cicatrices (las cuáles pasan a ser inapreciables). A pesar de ello, no todos los pacientes son candidatos a este tipo de intervención 


\section{Colecistectomía laparoscópica cuidados postoperatorios}

Vol. 3, núm. 3., (2019)

Nelson Ricardo Chacha Suscal; José Luis Campoverde Campoverde; Mayra Vanessa García Contreras; Luis Horacio Holguín Carranza; Paulina Graciela Vallejo Campuzano; Nelson Oswaldo Zavala Farfán

por lo que la técnica utilizada deberá ser consensuada entre cirujano y paciente según las características de este. De no ser posible no hay de qué preocuparse, pues la cirugía convencional de extracción de vesícula es también segura y efectiva.

Por lo tanto, una vez en el hogar, es importante que el paciente cumpla una serie de recomendaciones con el fin de reducir situaciones adversas y lograr la recuperación en forma más efectiva y eficiente. De allí, que una vez que el paciente culmine su estadía en la clínica u hospital, puede llegar a sufrir molestias en la incisión durante las dos primeras semanas aproximadamente, alargándose la recuperación total de 4 a 8 semanas tras la intervención Es así como, Dávila (ob.cit), cita una serie de recomendaciones postoperatorias una vez que el paciente es intervenido mediante la técnica de colecistectomía laparoscópica de vesícula biliar, entre ellas se encuentran las siguientes:

En los primeros días, el paciente debe tener una dieta líquida o tipo puré, tener cuidado para no moverse excesivamente, garantizando así que la herida quirúrgica cicatrice bien. Luego la alimentación será normal, sin embargo, se recomienda que sea baja en grasas, por lo que el paciente evite ingerir alimentos como embutidos, frituras, mayonesa, salsas, entre otros.

Asimismo, puede incluir en su dieta, el consumo de carnes bajas en grasa como pescados, pollos sin piel y pavo. Frutas excepto coco, granos cocidos. Puede ingerir granos integrales como avena, arroz, pan y pasta integral, leches y yogures descremados, quesos blancos como ricota, requesón light.

Después de la cirugía para extraer la vesícula se indica reposo inicial, pero tan pronto como se pueda levantar, después de 1 a 2 días, es posible hacer pequeñas caminatas y actividades sin esfuerzo. En el caso de la cirugía laparoscópica el paciente podrá volver al trabajo, manejar y realizar ejercicios suaves en 1 semana, en el caso de la cirugía convencional tendrá que esperar 2 semanas.

Es también importante que el paciente evite quedarse mucho tiempo sentado o acostado y, por eso, debe caminar varias veces al día. Aunque no hay una dieta establecida por extirpación de la 


\section{Colecistectomía laparoscópica cuidados postoperatorios}

Vol. 3, núm. 3., (2019)

Nelson Ricardo Chacha Suscal; José Luis Campoverde Campoverde; Mayra Vanessa García Contreras; Luis Horacio Holguín Carranza; Paulina Graciela Vallejo Campuzano; Nelson Oswaldo Zavala Farfán

vesícula biliar, los siguientes consejos pueden ayudar a minimizar los problemas con la diarrea después de que te hayan extirpado la vesícula biliar:

Modera tu consumo de grasa: Evita los alimentos altos en grasa, los alimentos fritos y grasosos, y las salsas con grasa durante al menos una semana después de la cirugía. En su lugar, escoge alimentos sin grasa o bajos en grasa. Los alimentos bajos en grasa son aquellos que no tienen más de 3 gramos de grasa por porción. Revisa las etiquetas y respeta el tamaño de la porción indicada.

Aumenta la fibra en tu dieta: Esto puede ayudar a normalizar las deposiciones. Agrega fibra soluble, como avena y cebada, a tu dieta. Pero asegúrate de aumentar la cantidad de fibra lentamente, por ejemplo, durante varias semanas, porque demasiada fibra al principio puede empeorar los gases y los calambres.

Come porciones pequeñas con más frecuencia: Esto puede asegurar una mejor mezcla con la bilis disponible. Una comida saludable debe incluir pequeñas cantidades de proteína magra, como aves de corral, pescado o productos lácteos sin grasa, junto con verduras, frutas y granos enteros.

También puedes tratar de limitar los alimentos que tienden a empeorar la diarrea, incluidos los siguientes: Cafeína. Productos lácteos. Alimentos muy dulces. El médico puede recomendar medicamentos, como loperamida (Imodium A-D), que retarda el movimiento intestinal, o medicamentos que disminuyen el efecto laxante de la bilis, como la colestiramina (Prevalite). Además, puede sugerirte que tomar un multivitamínico para compensar la mala absorción de vitaminas liposolubles.

\section{Conclusiones.}

La realización de la revisión bibliográfica, se convierte en una etapa fundamental para la construcción del cuerpo teórico del artículo, pues, de sus argumentaciones el investigador logra elaborar nuevos criterios esenciales que le proyectan al lector una comprensión simplificada del fenómeno en estudio. 


\section{Colecistectomía laparoscópica cuidados postoperatorios}

Vol. 3, núm. 3., (2019)

Nelson Ricardo Chacha Suscal; José Luis Campoverde Campoverde; Mayra Vanessa García Contreras; Luis Horacio Holguín Carranza; Paulina Graciela Vallejo Campuzano; Nelson

Oswaldo Zavala Farfán

El aparato digestivo como los demás sistemas que constituyen al ser humano, cumple funciones importantes para la realización de las actividades diarias, laborales y personales, son esenciales para tener calidad de vida. Es allí, donde el hígado como productor de bilis un líquido verdeamarillento, espeso y viscoso, que ayuda a la digestión al facilitar la absorción de colesterol, de otras grasas y vitaminas liposolubles desde el intestino. La bilis también ayuda a eliminar ciertos productos de desecho del organismo (principalmente la bilirrubina y el exceso de colesterol), así como subproductos de la descomposición de los fármacos.

Por ello, las vías biliares están compuestas por pequeños conductos que transportan la bilis desde el hígado hasta la vesícula biliar, y después al intestino delgado. La vesícula biliar es un órgano pequeño, con forma de pera, situado bajo el hígado. Almacena la bilis. Cuando el organismo necesita bilis, como cuando una persona come, la vesícula biliar se contrae y empuja la bilis a través de las vías biliares hacia el interior del intestino delgado. Pero la presencia de cálculos biliares que son depósitos duros de líquido digestivo que pueden formarse en la vesícula biliar, dependiendo de su tamaño puede variar desde un grano de arena hasta el de una pelota de golf. Algunas personas presentan un único cálculo biliar, mientras que otras desarrollan muchos cálculos biliares al mismo tiempo. Las personas que experimentan síntomas a causa de los cálculos biliares normalmente requieren una cirugía de extracción de la vesícula biliar. Los cálculos biliares que no provocan signos ni síntomas generalmente no requieren tratamiento.

En consecuencia, los diferentes síntomas que experimentan la persona, como consecuencia de los cálculos biliares, conduce a la necesidad de recibir tratamiento, así como la respectiva extirpación de la vesícula biliar, como recomendación final, todo depende de las condiciones del órgano, encontrado durante la fase de diagnóstico. Es allí, donde el médico tratante, recomienda la realización de una colecistectomía laparoscópica, instrumentos quirúrgicos especiales y una pequeña videocámara se introducen en tu abdomen a través de cuatro incisiones. El abdomen se infla con dióxido de carbono para darles a los instrumentos quirúrgicos más lugar para trabaja.

Por lo tanto, una vez realizada la intervención quirúrgica de una colecistectomía laparoscópica, los cuidados postoperatorios recomendados para una recuperación efectiva, se llevan a cabo durante 


\section{Colecistectomía laparoscópica cuidados postoperatorios}

Vol. 3, núm. 3., (2019)

Nelson Ricardo Chacha Suscal; José Luis Campoverde Campoverde; Mayra Vanessa García Contreras; Luis Horacio Holguín Carranza; Paulina Graciela Vallejo Campuzano; Nelson Oswaldo Zavala Farfán

un lapso de 6 semanas. Sin embargo, durante la misma puede el paciente tener algunos síntomas a medida que se restablece entre ellos: diarrea, vómito, dolor abdominal entre otros. En cuanto a la atención o cuidado que debe cumplirse se encuentra la realización de una dieta baja en grasa, aumento del consumo de granos integrales, yogurt, quesos descremados como el requesón, asimismo, impedir el consumo de granos cocidos, ayudarse con la alimentación de pescado, carne magra, pavo y pollo. De igual manera, evitar el desplazamiento de movimientos con alta frecuencia para ayudar al proceso de cicatrización de una forma normal, es decir, evitar ejercicios que logren generar hinchazón en el abdomen.

\section{Bibliografía.}

Arias, F. (2012). El Proyecto de INvestigación. Caracas: Episteme.

Dávila, A. (2017). Diagnóstico por Ecografía. Marban, 36-48.

Matos, C. (2016). Cirugía Laparoscópica de Vesícula Biliar. Cigna, 25-32.

Rangel, M. (2018). Dieta para después de la Extracción de la Vesícula. MayoClinc, 8-16.

Rojas, P. (2017). Colecistitis. MayoClinic, 19-27.

Sandoval, B. (2015). Esquema de Elaboración del Proyecto de Investigación. Caracas: Greco.

Ventura, S. (2018). El Proyecto de Investigación. Caracas: Oasis.

$$
\text { (c) }(1)(2)(2)
$$

RECONOCIMIENTO-NOCOMERCIAL-COMPARTIRIGUAL

CC BY-NC-SA

ESTA LICENCIA PERMITE A OTROS ENTREMEZCLAR, AJUSTAR Y CONSTRUIR A PARTIR DE SU OBRA CON FINES NO

COMERCIALES, SIEMPRE Y CUANDO LE RECONOZCAN LA AUTORÍA Y SUS NUEVAS CREACIONES ESTÉN BAJO UNA LICENCIA CON LOS MISMOS TÉRMINOS. 